\title{
ADMISSIBLE AND SINGULAR TRANSLATES OF MEASURES ON VECTOR SPACES
}

\author{
BY
}

ALAN GLEIT AND JOEL ZINN

\begin{abstract}
We provide a general setting for studying admissible and singular translates of measures on linear spaces. We apply our results to measures on $D[0,1]$. Further, we show that in many cases convex, balanced, bounded, and complete subsets of the admissible translates are compact. In addition, we generalize Sudakov's theorem on the characterization of certain quasi-invariant sets to separable reflexive spaces which have the Central Limit Property.
\end{abstract}

Introduction. It is the purpose of this paper to provide a general setting for studying admissible and singular translates of measures on linear spaces. In [9] Sudakov studied admissible translates of measures on a separable Hilbert space by exploiting Hilbert-Schmidt operators. These operators were also considered by Dudley [1] but are not available in a more general context. However, by considering maps between spaces one can obtain analogues of those operators. Based on our results we would conjecture the following (cf. Corollary 1.8 below):

Let $X$ be a locally convex linear topological space. Let $\mu$ be a positive Borel measure on $X$ for which the set of admissible translates forms a subspace of $X$. Then there exists auxiliary spaces $Y_{\alpha}$ and "compact" maps $T_{\alpha}: Y_{\alpha} \rightarrow X$, such that the set of admissible translates of $\mu$ is given by $\bigcap_{\alpha} T_{\alpha}\left(Y_{\alpha}\right)$.

The organization of the paper is as follows. The first section contains general theorems in which properties of certain auxiliary spaces and maps are discussed. In $\$ 2$ we show that in many cases a convex, balanced, complete, and bounded subset of the admissible translates is compact. In $\S 3$ we extend Sudakov's theorem on the characterization of certain quasi-invariant sets to separable reflexive Banach spaces with the Central Limit Property. These include $l_{p}$ and separable $L^{p}(m)$-spaces, $2 \leqslant p<\infty$. In $\S 4$ we apply the techniques of $\S \S 1$ and 2 to obtain similar results in the nontopological vector space $D[0,1]$. To the best of our knowledge these are the first general results on admissible translates for measures on $D[0,1]$.

Received by the editors March 8, 1974 and, in revised form, September 9, 1974 and June 23, 1975.

AMS (MOS) subject classifications (1970). Primary 28A40; Secondary 46A05, 60B05.

Key words and phrases. Admissible translates, singular translates, Skorokhod topology on $D[0,1]$, measures on vector spaces, quasi-invariant sets.

Copyright $\bigcirc$ 1976, American Mathematical Society 
We should like to thank the referee of an earlier draft of this paper for his helpful comments. In particular, he suggested generalizing Sudakov's theorem and this led to $\S 3$.

1. General theorems. Let $\mu$ be a positive Borel measure on a locally convex topological vector space (lctvs) $X$. For $x \in X$ we define $\mu_{x}$ to be the "translation of $\mu$ by $x$ "; more precisely, $\mu_{x}(E)=\mu(E-x)$ for all Borel sets $E$. For measures $\gamma$ and $\tau$ on $X, \gamma<<\tau$ (resp., $\gamma \perp \tau$ ) will mean $\gamma$ is absolutely continuous with respect to $\tau$ (resp., singular to $\tau$ ). If $\gamma<<\tau$ and $\tau<<\gamma$ we say $\gamma$ is equivalent to $\tau$ and write $\gamma \sim \tau$. We now define

$$
A_{\mu}=\left\{x \in X: \mu_{x} \sim \mu\right\} \quad \text { and } S_{\mu}=\left\{x \in X: \mu_{x} \perp \mu\right\} \text {. }
$$

The elements of $A_{\mu}$ (resp., $S_{\mu}$ ) will be called admissible (resp., singular) translates (of $\mu$ ).

Let $Y=$ linear span of the support of $\mu$ and let $\nu=\left.\mu\right|_{Y}$. Since our main concern is the study of $A_{\mu}$ and $\left(S_{\mu}\right)^{c}$, and since $A_{\mu}=A_{\nu}$ and $S_{\mu}=S_{\nu} \cup(X \backslash Y)$, we see that there is no loss of generality in assuming that $X$ is the linear span of the support of $\mu$ when we do. Further, a measure $\mu$ is said to be separable if the support of $\mu$ is separable.

In this section we will obtain results on the sets $A_{\mu}$ and $\left(S_{\mu}\right)^{c}$ and on some of their subsets. To do this we introduce some auxiliary spaces and define certain maps. Let $E$ be a Borel set in $X$ of positive $\mu$-measure. We write

$$
\|x *\|_{\mu, E}^{2}=\int_{E}|x *(x)|^{2} \mu(d x)
$$

for each $x^{*} \in X^{*}$, where we assume that conditions have been placed on $\mu$ and/or $E$ so that $\|x *\|_{\mu, E}<\infty$ for each $x^{*} \in X^{*}$. We let $V_{\mu, E}$ be the completion of $X^{*}$ in the seminorm $\|-\|_{\mu, E}$ and $N$ be the elements $n$ in $V_{\mu, E}$ with $\|n\|_{\mu, E}=0$. Finally, let $T_{\mu, E}: X^{*} \rightarrow V_{\mu, E} / N$ be the composition of the natural inclusion of $X^{*}$ into $V_{\mu, E}$ with the natural projection of $V_{\mu, E}$ onto $V_{\mu, E} / N$. We will let $\hat{V}_{\mu, E}$ denote $V_{\mu, E} / N$. If $E=X$, we will suppress the reference to $E$ in all maps and spaces.

In the remainder of this paper, we will use $k$ to denote the natural embedding of any normed space in its bidual.

First, a well-known lemma.

LEMMA 1.1. Let $T: A \rightarrow B$ be a weakly continuous map between lctvs's and let $T^{*}: B^{*} \rightarrow A^{*}$ be its adjoint. $a^{*} \in A^{*}$ is not in $T^{*}\left(B^{*}\right)$ iff there exists a net $\left\{a_{\alpha}\right\} \subseteq A$ satisfying:

(i) $a^{*}\left(a_{\alpha}\right)=1$ for all $\alpha$, and

(ii) $T\left(a_{\alpha}\right) \rightarrow 0$.

If $B$ is metrizable, we may choose a sequence satisfying (i) and (ii). 
Proof. Suppose $a^{*} \notin T^{*}\left(B^{*}\right)$. If $a^{*}$ does not annihilate the null space of $T$, then clearly we may choose a constant net. If $a^{*}$ annihilates the null space of $T$ and $a^{*} \notin T^{*}\left(B^{*}\right)$, then define $\overline{a^{*}}$ on $T(A)$ by: $\overline{a^{*}}(T a)=a^{*}(a)$. If $\overline{a^{*}}$ were continuous on $T(A)$, it could be extended to an element $b^{*} \in B^{*}[8$, Corollary, p. 29]. But then clearly $T^{*}\left(b^{*}\right)=a^{*}$ and so $\overline{a^{*}}$ is not continuous on $T(A)$. The conclusion follows immediately. The converse is obvious.

THEOREM 1.2. Let $\mu$ be a separable positive Borel measure on a normed linear space, and let $X$ be the closed linear span of the support of $\mu$ Suppose also that $\int_{X}\left\|_{x}\right\|^{2} \mu(d x)=K^{2}<\infty$. Then $V_{\mu}$ is well defined, $T_{\mu}$ and $T_{\mu}^{*}$ are continuous, compact operators and $T_{\mu}^{*}\left(V_{\mu}^{*}\right) \supseteq\left(S_{\mu}\right)^{c}$.

Proof. We first note that $\|x *\|_{\mu} \leqslant K \cdot\|x *\|$, which implies that $V_{\mu}$ is well defined and $T_{\mu}$ is continuous. Next, we show that $T_{\mu}$ is compact. As both $X^{*}$ and $V_{\mu}$ are normed spaces, it suffices to show that every bounded sequence in $X^{*}$ has a convergent subsequence in $V_{\mu}$. Let $\left\{x_{n}^{*}\right\} \subseteq X^{*}$ satisfy $\left\|x_{n}^{*}\right\| \leqslant M$. As $X$ is separable, there exists a subsequence of $\left\{x_{n}^{*}\right\}$ converging weak* to an element $x^{*} \in X^{*}$. By the Dominated Convergence Theorem, this subsequence converges to $x^{*}$ with respect to $\|-\|_{\mu}$ and so $T_{\mu}$ is compact.

Now let $c \in\left(S_{\mu}\right)^{c}$ and let $\alpha$ be the natural imbedding of $X$ in its bidual. If $K(c) \notin T_{\mu}^{*}\left(V_{\mu}^{*}\right)$, then by Lemma 1.1 there is a sequence $\left\{x_{n}^{*}\right\} \subseteq X^{*}$ satisfying $\left\|T_{\mu}\left(x_{n}^{*}\right)\right\|_{\mu} \rightarrow 0$ and $1=\kappa(c)\left(x_{n}^{*}\right)=x_{n}^{*}(c)$ for all $n$. But

$$
\mu\left[x \in X:\left|x_{n}^{*}(x)\right| \geqslant \epsilon\right] \leqslant \frac{1}{\epsilon^{2}} \int_{X}\left|x_{n}^{*}(x)\right|^{2} \mu(d x)=\frac{1}{\epsilon^{2}}\left\|T_{\mu}\left(x_{n}^{*}\right)\right\|_{\mu}^{2}
$$

and the latter tends to zero. Hence by going to a subsequence and re-indexing, we may assume $x_{n}^{*} \rightarrow 0$ a.e. $[\mu]$. On the other hand,

$$
\begin{aligned}
\mu_{c}\left[x \in X: x_{n}^{*}(x) \rightarrow 0\right] & =\mu\left[x \in X: x_{n}^{*}(x+c) \rightarrow 0\right] \\
& =\mu\left[x \in X: x_{n}^{*}(x) \rightarrow-1\right] .
\end{aligned}
$$

From above, the measure of the latter is zero. Hence $\mu \perp \mu_{c}$, a contradiction.

Corollary 13. Let $\mu$ be a separable positive, $\sigma$-finite Borel measure on a normed linear space, and let $X$ be the closed linear span of the support of $\mu$ Then there exists a (finite) measure $\nu$ equivalent to $\mu$ such that:

$$
T_{\nu}^{*}\left(V_{\nu}^{*}\right) \supseteq\left(S_{\mu}\right)^{c} \text { and both } T_{\nu} \text { and } T_{\nu}^{*} \text { are compact. }
$$

Proof. As $\mu$ is $\sigma$-finite, it is equivalent to a finite measure $m$. The latter measure is equivalent to $d \nu=\exp (-\|x\|) d m$. Clearly, $\int\|x\| \nu(d x)<\infty, S_{\mu}=S_{\nu}$ and $A_{\mu}=A_{\nu}$.

For an lctvs $X$ we may topologize $X^{*}$ in many ways. The strong topology 
on $X^{*}$, denoted by $\beta$, is the topology of uniform convergence on each (weakly) bounded set in $X$. The weak* topology on $X^{*}$ is the topology of pointwise convergence on elements of $X$. The bidual $X^{* *}$ of $X$ is the dual of $\left(X^{*}, \beta\right)$ in the strong topology. For the purposes of this paper, $X$ is said to be allowable if: Every strongly bounded set in $X^{*}$ is equicontinuous. Each bornological space is allowable $[8$, Proposition 9, p. 83] and so each metric lctvs is allowable [8, Proposition 8, p. 82]. Also, each barrelled space is allowable [8, Corollary 1, p. 66] and so inductive limits of Fréchet spaces are allowable [8, Proposition 6, p. 81]. We note that for allowable spaces, the natural imbedding of $X$ into its bidual $X^{* *}$ is an isomorphism [8, Proposition 2, p. 71] and so we may identify $X$ with its image in $X^{* *}$.

The next theorem and its corollary might be of use in proving local results about $A_{\mu}$.

THEOREM 1.4. Let $X$ be a separable allowable lctvs. Let $\mu$ be a positive Borel measure on $X$. Let $0 \in C \subseteq A_{\mu}$. Suppose there exist measurable sets $U$ and $F$ such that:

(1) $\mu(U)>0$,

(2) $F \supseteq U \cup(U-C)$,

(3) $\mu(F)<\infty$,

(4) $F$ is weakly bounded.

Let $T=T_{\mu, F}$. Then

(a) $T$ of a strongly bounded set is relatively compact,

(b) $T^{*}$ of a strongly bounded set is relatively compact in $X^{* *}$, and

(c) $C$ is in the image of $T^{*}$.

We note that properties (3) and (4) for the set $F$ are sufficient to guarantee that $\|x\|_{\mu}$ exists and that $\hat{V}_{\mu, F}$ is nontrivial. We first prove a technical lemma.

Lemma 1.5. Suppose that $X, \mu$ and $T$ are as above. Suppose $\left\{x_{\alpha}^{*}\right\} \subseteq X^{*}$ and $x^{*} \in X^{*}$ satisfy:

(1) $x_{\alpha}^{*} \rightarrow x^{*}$ weak*, and

(2) $\left\{x_{\alpha}^{*}, x^{*}\right\}$ are contained in a strongly bounded set $B$.

Then $T\left(x_{\alpha}^{*}\right) \rightarrow T\left(x^{*}\right)$.

Proof. If not, there is an $\epsilon>0$ and a subnet $\left\{x_{\beta}^{*}\right\}$ such that $\| T\left(x_{\beta}^{*}\right)-$ $T\left(x^{*}\right) \| \geqslant \epsilon$. As $x_{\beta}^{*} \rightarrow x^{*}$ weak*, we may find a cofinal sequence $x_{\beta_{n}}^{*} \rightarrow x^{*}$ weak*, since the weak* topology is metrizable on equicontinuous sets. Also, there is a $K<\infty$ with $\left|x_{\beta_{n}}^{*}(x)\right|<K$ for each $\beta_{n}$ and each $x \in F$ (since $B$ is strongly bounded). The Dominated Convergence Theorem then asserts that $\left\|x_{\beta_{n}}^{*}-x\right\|_{\mu, F}$ $\rightarrow 0$ and so $\left\|T\left(x_{\beta_{n}}^{*}\right)-T\left(x^{*}\right)\right\| \rightarrow 0$, a contradiction.

ProOf OF THEOREM 1.4. We first show that $T$ is continuous. Let $x_{\alpha}^{*} \rightarrow$ 
$x^{*}$ strongly. Then $\left\{x_{\alpha}^{*}, x^{*}\right\}$ is strongly bounded and $x_{\alpha}^{*} \rightarrow x^{*}$ weak*. By the lemma, $T\left(x_{\alpha}^{*}\right) \rightarrow T\left(x^{*}\right)$ and so $T$ is continuous. We next show that $T$ of any strongly bounded set $B$ is relatively compact in $\hat{V}_{\mu, F}$. In fact we will show that $T(\bar{B})$ is compact. Let $\left\{x_{\alpha}^{*}\right\} \subseteq B$. Then $\bar{B}$, the closure of $B$, is weak* compact $\left[8\right.$, Corollary 1, p. 62]. Thus there is a subnet $\left\{x_{\beta}^{*}\right\}$ and $x^{*} \in \bar{B}$ with $x_{\beta}^{*} \rightarrow x^{*}$ weak*. Thus $T\left(x_{\beta}^{*}\right) \rightarrow T\left(x^{*}\right)$ by the lemma and so $T$ has the property claimed. Hence $T^{*}$ of any bounded set is contained in a compact set in the bidual $X^{* *}$ [8, Proposition 5, p. 153].

Now let $c \in C$ and suppose $c \notin T^{*}\left(\hat{V}_{\mu, F}^{*}\right)$. By Lemma 1.1 there is a sequence $\left\{x_{n}^{*}\right\} \subseteq X^{*}$ such that $\left\|T\left(x_{n}^{*}\right)\right\| \rightarrow 0$ and $x_{n}^{*}(c)=1$. As $\left\|T\left(x_{n}^{*}\right)\right\|=$ $\left\|x_{n}^{*}\right\|_{\mu}$, we have

$$
\mu\left[x \in F:\left|x_{n}^{*}(x)\right| \geqslant \epsilon\right] \leqslant \frac{1}{\epsilon^{2}} \int_{F}\left|x_{n}^{*}(x)\right|^{2} \mu(d x)=\frac{1}{\epsilon^{2}}\left\|T\left(x_{n}^{*}\right)\right\|^{2}
$$

and the latter tends to zero. Hence by going to a subsequence and re-indexing, we may assume $x_{n}^{*} \rightarrow 0$ a.e. $[\mu]$ on $F$. Let $E=\left\{x \in F: x_{n}^{*}(x) \rightarrow 0\right\}$. Then $\mu(E)=\mu(F)$. Hence $\mu(U \cap E)>0$. On the other hand

$$
\begin{aligned}
\mu_{c}(U \cap E) & =\mu_{c}\left[x \in U: x_{n}^{*}(x) \rightarrow 0\right] \\
& =\mu\left[x \in U-c: x_{n}^{*}(x+c) \rightarrow 0\right] \\
& \leqslant \mu\left[x \in F: x_{n}^{*}(x) \rightarrow-1\right]=0 .
\end{aligned}
$$

However this contradicts the fact that $\mu_{c}$ is equivalent to $\mu$. Hence $C \subseteq$ $T^{*}\left(\hat{V}_{\mu, F}^{*}\right)$.

COROllary 1.6. Let $\mu$ be a separable, positive, Borel measure on a normed linear space. Let $0 \in C \subseteq A_{\mu}$. Suppose there exists a measurable set $U$ and a measurable set $F \supseteq U \cup(U-C)$ such that:

(1') $\mu(U)>0$,

(2') $0<\int_{F}\|x\|^{2} \mu(d x)<\infty$.

Define $T$ as in Theorem 1.4. Then $T$ and $T^{*}$ are compact and $C$ is in the range of $T^{*}$.

Proof. Property ( $\left.2^{\prime}\right)$ of $U$ listed above is sufficient to guarantee that $\left\|x^{*}\right\|_{\mu}<\infty$ for each $x^{*} \in X^{*}$. The only other use of the stronger properties of $U$ in Theorem 1.4 was in the use of the Dominated Convergence Theorem in the proof of Lemma 1.5. Here we may use the bound $\left|x^{*}(x)\right| \leqslant K\|x\|$ for all $x^{*}$ in a strongly bounded set and use (2').

The next theorem will be useful in proving global results about $A_{\mu}$.

THEOREM 1.7. Let $\mu$ be a finite, positive, outer regular Borel measure on an lctvs $Y$. Suppose there exists a compact set $K$ of positive $\mu$-measure. Let $Z$ 
be the closed linear span of $K$. Let $X$ be any allowable closed subspace satisfying $Y \supseteq X \supseteq Z$. Let $T=T_{\mu, K}$. Then:

(a) $T$ of a strongly bounded set is relatively compact,

(b) $T^{*}$ of a strongly bounded set is relatively compact, and

(c) the largest subspace of $A_{\mu}$ is contained in the range of $T^{*}$.

Proof. By hypothesis every strongly bounded set, $B$, in $X^{*}$ is equicontinuous. Hence $B$ is equicontinuous on $K$ and so is a precompact subset of $C(K)$. So any net in $B$ has a cofinal subsequence which converges uniformly on $K$. Therefore $T(B)$ is relatively compact by the Dominated Convergence Theorem. Again by [8, Proposition 5, p. 153] $T^{*}$ has the same property.

Now let $M$ denote the largest subspace of $A_{\mu}$. By Proposition 1 in [11] we have $M \subseteq Z$. Note that in the proof of that proposition regularity and tightness are not needed for Borel subgroups. Now suppose that $c \in M \subseteq X^{* *}$ is not in $T^{*}\left(V_{\mu, K}^{*}\right)$. Then by Lemma 1.1 there exists a sequence $\left\{x_{n}^{*}\right\} \subseteq X^{*}$ such that $x_{n}^{*}(c)=1$ for all $n$ and $T\left(x_{n}^{*}\right) \rightarrow 0$. As before we have $\mu\left[x \in X: x_{n}^{*}(x) \rightarrow 0\right]$ $>0$. Hence for $|\lambda| \leqslant 1$,

$$
0<\mu_{\lambda c}\left[x \in X: x_{n}^{*}(x) \rightarrow 0\right]=\mu\left[x \in X: x_{n}^{*}(x) \rightarrow-\lambda\right] .
$$

But the sets $E_{\lambda}=\left\{x \in X: x_{n}^{*}(x) \rightarrow-\lambda\right\}$ are pairwise disjoint, which contradicts the finiteness of $\mu$.

COROLlaRy 1.8. Let $\mu$ be finite, positive, outer regular Borel measure on an allowable lctvs. Let $L=$ the largest subspace of $A_{\mu}$. Then

$$
L \subseteq \bigcap\left\{\text { Range } T_{\mu, K}^{*}: K \text { compact, } \mu(K)>0\right\} .
$$

2. Application to compactness in lctvs. In this section we shall show that in many cases a complete, convex, balanced, bounded set, which is contained in $A_{\mu}$, is compact. Actually, more is proved in particular cases. First some technical lemmas.

LEMMA 2.1. Suppose $C$ is a closed, starlike set in a normed linear space. Suppose also that $C$ contains no ray and $C \cap\{x:\|x\| \leqslant 1\}$ is compact. Then $C$ is bounded and hence compact.

LEMMA 2.2 [1]. Let $T: A \rightarrow B$ be a continuous linear map from a Banach space $A$ into an lctvs $B$. Suppose that $D$ is a convex, balanced, bounded, complete set which is contained in the range of $T$. Then $D=T(M)$ for some bounded set $M$.

THEOREM 2.3. Let $\mu$ be a separable, positive Borel measure on a normed linear space, and let $X$ be the closed linear span of the support of $\mu$ Suppose, 
also, that $\int\|x\|^{2} \mu(d x)<\infty$. Let $C$ be a convex, balanced, complete subset of $\left(S_{\mu}\right)^{c}$. Then $C=W+T_{\mu}^{*}(M)$, where $W$ is a finite-dimensional subspace and $M$ is a bounded set in $V_{\mu}^{*}$. Hence, if $C$ were bounded, it would be compact.

Proof. Follows from the lemmas as in [1].

We should like to compare our results with those of Xia. A special case of one of his results is the following.

THEOREM 2.4 [10, THEOREM 3.1.13]. Let $\mu$ be a positive regular Borel measure on an lctvs $X$. Let $C$ be a convex, balanced, bounded subset of $A_{\mu}$. Suppose that the linear span of $C$ is second category in a stronger topology than the relative topology of $X$. Finally, suppose that there exists a compact set $K$ $\subseteq X$, which has finite, positive $\mu$-measure. Then $C$ is contained in a compact set.

We note that if $C$ is complete, then the Minkowski gauge (of $C$ ) on the span of $C$ makes the latter into a Banach space. Now the topology on the span of $C$ induced by the Minkowski gauge (of $C$ ) is stronger than the relative topology. Hence we have the following corollary.

COROLlaRY 2.5. Let $\mu$ be a positive, regular Borel measure on an lctvs $X$. Suppose that $C \subseteq A_{\mu}$ is convex, balanced, bounded and complete. Suppose also that there exists a compact set in $X$ of finite, positive $\mu$-measure. Then $C$ is compact.

We should now like to prove a result similar to Corollary 2.5 using the techniques developed above. We shall add more assumptions to the space and change slightly the assumptions on the measure $\mu$. In return, as we have seen in $\S 1$, we have $C$ is the image of a bounded set under a "computable" compact map from a "computable" Banach space. In many cases, such as when the Banach space $X$ has the weak* topology (and so all complete and bounded sets are compact), our results are much stronger than his. In addition, Xia's original theorem is stated for topological groups, and the continuity of addition is used heavily. However, we shall show in $\S 4$ that our techniques are sometimes applicable in cases in which addition is not continuous.

THEOREM 2.6. Under the hypotheses of Theorem 1.4 and the additional assumption that $C$ is convex, balanced, bounded and complete, we have that $C$ is compact. Moreover, $C$ is of the form $T^{*}(M)$ for some bounded set $M$.

Proof. Follows immediately from the conclusions of Theorem 1.4 and Lemma 2.2.

THEOREM 2.7. Under the hypotheses of Theorem 1.7 and the additional assumption that $C$ is a complete, bounded, convex, balanced set in $A_{\mu}$, we have 
that $C$ is compact. Moreover, $C=T^{*}(M)$ for some bounded set $M$.

3. A generalization of a theorem of Sudakov. In this section we shall give necessary and sufficient conditions for a closed, balanced, and convex set $C$ in a separable $L^{p}(m)$-space or in $l^{p}, \infty>p \geqslant 2$, to be a subset of the admissible translates of a probability measure $\mu$. Sudakov [9] showed that for $p=2, C \varsigma$ $A_{\mu}$ for some $\mu$ if and only if $C=W+S(M)$ where $W$ is a finite-dimensional subspace, $M$ is bounded and $S$ is a Hilbert-Schmidt operator. Our Theorem 2.3 provides the necessary condition; for sufficiency, we must first analyze the map $T_{\mu}$ in more detail. First, we recall [7] that a map $T: A \rightarrow B$ between normed spaces is $p$-absolutely summing if there exists a finite constant $K$ such that for all finite sequences $\left\{a_{i}\right\} \subseteq A$ we have

$$
\sum_{i}\left\|T a_{i}\right\|^{p} \leqslant K \sup \left\{\sum\left|b^{*}\left(a_{i}\right)\right|^{p}:\left\|b^{*}\right\| \leqslant 1, b^{*} \in B^{*}\right\} .
$$

LEMma 3.1. Let $\mu$ be a finite, positive Borel measure on $X$ with $\int\|x\|^{2} d \mu$ $<\infty$. Let $T_{\mu}: X^{*} \rightarrow V_{\mu}$ be as in $\$ 2$. Then

(1) $V_{\mu}$ is a separable Hilbert space,

(2) $T_{\mu}^{*}: V_{\mu}^{*} \rightarrow \kappa(X)$ and so $T_{\mu}=\left(\kappa^{-1} T_{\mu}^{*}\right)^{*}$,

(3) $T_{\mu}$ is 2-absolutely summing.

Proof. Clearly $V_{\mu}$ is a sub-Hilbert space of $L^{2}(\mu)$. The Bounded Convergence Theorem and the fact that $X^{*}$ is weak* separable imply that $V_{\mu}$ is separable. Identifying $V_{\mu}^{*}$ with $V_{\mu}$, we easily compute

$$
T_{\mu}^{*} T_{\mu}\left(x^{*}\right)=\int_{X} x^{*}(x) x d \mu(x) .
$$

As

$$
\int_{X}\left\|x^{*}(x) x\right\| d \mu \leqslant\|x *\| \int_{X}\|x\|^{2} d \mu<\infty,
$$

we have that $T_{\mu}^{*} T_{\mu}: X^{*} \rightarrow \kappa(X)$. Assertion (2) follows easily. If $\mu=\delta(0)$, then (3) is trivial. Otherwise define a positive measure $\nu$ on the unit sphere $S$ of $X$ by $m(B)=\int_{B^{\prime}}\|x\|^{2} d \mu(x)$ where $B^{\prime}=\{x: x /\|x\| \in B\}$ for all Borel sets $B$. Clearly $\left\|T_{\mu} x^{*}\right\|^{2}=\int_{S}\left|x^{*}(\theta)\right|^{2} d m(\theta)$ and so $T_{\mu}$ is 2-absolutely summing [7].

We now see that $T_{\mu}$ is more than "just" compact and this observation is the key to sufficiency for $L^{p}(m)$-spaces, $p \geqslant 2$. First some preparatory lemmas.

Lenima 3.2. Let $S$ map a separable Hilbert space $M$ to a normed space $X$. Suppose $S^{*}$ is 2-absolutely summing and let $W$ be a finite-dimensional subspace of $X$. Then there exists a map $\widetilde{S}$ from a separable Hilbert space $\widetilde{H}$ with $(\widetilde{S})^{*}$ 2-absolutely summing and Range $\widetilde{S} \supseteq$ (Range $S$ ) $\cup W$.

Proof. We may assume that $S(H) \cap W=\{0\}$. Let $l_{n}^{2}$ be a Hilbert space of dimension $n=\operatorname{dim} W$ and let $j: l_{n}^{2} \rightarrow W$ be a canonical isomorphism. Then 
$\widetilde{S}=S \oplus j: \widetilde{H}=\left(H \oplus l_{n}^{2}\right)_{2} \rightarrow X$ clearly satisfies all the conditions.

LEMma 3.3. Let $S$ be a compact map from a separable Hilbert space $H$ to a separable Banach space $X$. We identify $H$ with $H^{*}$. Suppose there is a Gaussian measure $\mu$ on $X$ satisfying

$$
S S^{*}\left(x^{*}\right)=\int x^{*}(x) x d \mu(x) .
$$

Then $A_{\mu}=$ Range( $(S)$.

Proof. Construct as in [5] a separable Hilbert space $K$ and a one-to-one mapping $i: X \rightarrow K$ such that the Borel sets of $K$ contained in $i(X)$ are the same as the images of the Borel sets of $X$. Then $\mu^{i}$, defined by $\mu^{i}(B)=\mu\left(i^{-1}(B \cap i(X))\right)$ for all Borel sets in $K$, defines a Gaussian measure on $K$. Then for $k^{*} \in K^{*}$,

$$
\begin{aligned}
\hat{\mu}^{i}\left(k^{*}\right)=\hat{\mu}\left(i^{*} k^{*}\right) & =\exp \left\{-1 / 2\left\langle S S^{*}\left(i^{*} k^{*}\right), i^{*} k^{*}\right\rangle\right\} \\
& =\exp \left\{-1 / 2\left\langle i S S^{*} i^{*} k^{*}, k^{*}\right\rangle_{K}\right\}
\end{aligned}
$$

where $\langle,\rangle_{K}$ is the inner product on $K$. Let $B=i S S^{*} i^{*}$. Writing, in canonical form, $B=\Sigma \lambda_{i} e_{i} \otimes e_{i}$, we have $A_{\mu i}=\left\{z: \Sigma\left|\left\langle z, e_{i}\right\rangle\right|^{2} / \lambda_{i}<\infty\right\}$ (see, for example, [5] or [11]). But then obviously

$$
A_{\mu^{i}}=\operatorname{Range} B^{1 / 2}=\operatorname{Range}\left(i S S^{*} i^{*}\right)^{1 / 2}=\operatorname{Range}\left((i S)(i S)^{*}\right)^{1 / 2} .
$$

By using the standard representation for compact operators between Hilbert spaces, one easily notes that $\operatorname{Range}(i S)=\operatorname{Range}\left((i S)(i S)^{*}\right)^{1 / 2}$. Hence $i\left(A_{\mu}\right)=$ $A_{\mu^{i}}=$ Range $(i S)=i($ Range $S)$.

We now define the Central Limit Property. Let $X$ be a separable Banach space and $\nu$ a probability measure on $X$ with $\int x v(d x)=0$ and $\int\|x\|^{2} \nu(d x)<\infty$. Let $y_{1}, y_{2}, \ldots$ be a sequence of independent $X$-valued random variables with distribution $\nu . \nu$ is said to satisfy the Central Limit Theorem is there exists a Gaussian probability measure $\mu$ on $X$ such that the distributions of $\left(y_{1}+\cdots+y_{n}\right) / \sqrt{n}$ converge $\|\cdot\|$-weakly to $\mu$. (For the definition of $\|\cdot\|$-weak convergence see [3].) $X$ satisfies the Central Limit Property if every such $\nu$ satisfies the Central Limit Theorem. This property immediately implies the following (in fact, is equivalent to it by [3]):

Whenever $\nu$ is a probability measure on $X$ with $\int x \nu(d x)=0$ and $\int\|x\|^{2} \nu(d x)<\infty$, there is a Gaussian probability measure $\mu$ on $X$ with

for each $x^{*} \in X^{*}$.

$$
\hat{\mu}\left(x^{*}\right)=\exp \left\{-\frac{1}{2} \int\left|x^{*}(x)\right|^{2} d \nu(x)\right\}
$$

We recall that Fortet and Mourier [2] have shown that $L^{p}[0,1]$ has the Central Limit Property for $2 \leqslant p<\infty$. As the Central Limit Property is clearly 
inherited by closed subspaces, we note that separable $L_{p}$-spaces, $2 \leqslant p<\infty$, satisfy this property and are always reflexive. (For a discussion of $L_{p}$-spaces, see [6].) Consequently, $l_{p}$ and separable $L^{p}(m)$ spaces satisfy the Central Limit Property.

We are now prepared for our generalization of Sudakov's theorem.

TheOREM 3.4. Let $C$ be a closed, convex, and balanced set in $X$, a separable reflexive Banach space having the Central Limit Property. Then $C$ is a subset of $A_{\mu}$ for some probability measure $\mu$ (i.e. $C$ is a quasi-invariant set) if and only if $C=W+S(M)$ where $W$ is a finite-dimensional subspace, $S$ is a (compact) map from a separable Hilbert space $H$ to $X, M$ is bounded in $H$, and $S^{*}$ is 2-absolutely summing.

Proof. If $C$ is a quasi-invariant set, the fact that $C$ is of the required form follows directly from Theorem 2.3 and Lemma 3.1. For the converse, assume $C=W+S(M)$ where $W, S$ and $M$ are as in the statement of the theorem. By Lemma 3.2, we may assume that $C$ is in the range of $S$. As $S^{*}$ is 2-absolutely summing, there is a probability measure $\nu$ on $U=$ unit ball of $X^{* *}$ $=X$ and a constant $K$ satisfying

$$
\left\|S^{*} x *\right\|^{2} \leqslant K \int_{U}\left|x^{*}(x)\right|^{2} d v(x)
$$

for all $x^{*} \in X^{*}$. By considering the probability measure $\bar{\nu}$ on $U$ defined by $\bar{\nu}(B)=(\nu(B)+\nu(-B)) / 2$ for Borel sets $B$, we may, without loss of generality, assume $\int_{U} x d \nu(x)=0$. Let $T=T_{\nu}$ be the natural inclusion of $X^{*}$ into its closure in $L^{2}(\nu)$.

We first claim that Range $S \subseteq$ Range $T^{*}$. Indeed, if $a \notin$ Range $T^{*}$, then by Lemma 1.1 , there exists $\left\{x_{n}^{*}\right\} \subseteq X^{*}$ such that $x_{n}^{*}(a)=1$ and $\left\|T x_{n}^{*}\right\|^{2} \rightarrow 0$. By (3.1), $\left\|S^{*} x_{n}^{*}\right\| \rightarrow 0$ and so, again by Lemma 1.1, $a \notin$ Range $S^{* *}=$ Range $S$. Hence $C \subseteq$ Range $T^{*}$.

By the Central Limit Property, we may find a Gaussian $\mu$ satisfying

$$
\hat{\mu}\left(x^{*}\right)=\exp \left\{-\frac{1}{2} \int\left|x^{*}(x)\right|^{2} d v(x)\right\}
$$

for $x^{*} \in X^{*}$. Consequently

$$
\hat{\mu}\left(x^{*}\right)=\exp \left\{-1 / 2\left\|T\left(x^{*}\right)\right\|^{2}\right\}
$$

for $x^{*} \in X^{*}$. As $\mu$ is Gaussian, we have $\left\langle T\left(y^{*}\right), T\left(x^{*}\right)\right\rangle=\int x^{*}(x) y^{*}(x) d \mu(x)$ for all $x^{*}, y^{*} \in X^{*}$ and so $T^{*} T\left(x^{*}\right)=\int x^{*}(x) x d \mu(x)$. Hence by Lemma 3.3, $C \subseteq$ Range $T^{*}=A_{\mu}$.

We should like to point out that for a Hilbert space the above result is identical to that of Sudakov since the Hilbert-Schmidt operators and the 2-absolutely summing operators are the same [7]. 
4. Application to compactness in $D[0,1]$. In $\$ 2$ we were able to apply the results of $\S 1$ to a large class of topological vector spaces. In this section we would like to show that the same techniques might be applied to spaces in which addition is not continuous. In particular, we shall consider the space $D=[0,1]$ consisting of functions on $[0,1]$ which are right continuous and have left-hand limits. The Skorokhod topology on this space is generated by the metric

$$
d(x, y)=\inf _{\lambda \in \Lambda} \max \left\{\|\lambda-\|_{\infty},\|x-y \circ \lambda\|_{\infty}\right\} \text { for } x, y \in D,
$$

where $\|-\|_{\infty}$ indicates the supremum norm, $\Lambda$ is the set of homeomorphisms of $[0,1]$ which fix 0 , and $I$ is the identity element of $\Lambda$, i.e., $I(s)=s$. $D$ is a topologically complete, separable metric space under $d(\cdot, \cdot)$.

Though $D$ is far from being a tvs, it does have many continuous linear functionals. In particular, the set of finite measures on $[0,1]$ which are continuous except for an atom at 1 are continuous. That is, if we let $Z$ denote this set of measures, then $x \rightarrow \int_{0}^{1} x(t) \nu(d t)$ is continuous for each $\nu$ in $Z$. The imbedding of $D$ into $Z^{*}$ is "nice". It is this imbedding which allows one to apply the methods of $\S 1$.

Proposition 4.1. Let the topology of $Z$ be given by the total variation norm. Let $\phi: D \rightarrow Z^{*}$ be the natural imbedding. Then for each $x \in D,\|\phi(x)\|$ $=\|x\|_{\infty}$. Hence $\phi$ is a closed map and $\left.\phi^{-1}\right|_{\phi(D)}$ is continuous.

Proof. Let $x \in D$.

$$
\begin{aligned}
\|\phi(x)\| & =\sup \{|\nu(x)|:\|\nu\| \leqslant 1, \nu \in Z\} \\
& \geqslant \sup \left\{\left|\frac{1}{\delta} \int_{s}^{s+\delta} x(t) d t\right|: 0 \leqslant s<1, \delta>0,0<s+\delta<1\right\} \\
& \geqslant \sup \{|x(s)|: 0 \leqslant s<1\}
\end{aligned}
$$

by right continuity. Hence $\|\phi(x)\| \geqslant\|x\|_{\infty}$ since the point mass at 1 is in $Z$. Clearly, $\|\phi(x)\| \leqslant\|x\|_{\infty}$. $\phi$ is closed since $D$ with the supremum norm is complete. Finally, $\left.\phi^{-1}\right|_{\phi(D)}$ is continuous since $d(x, y) \leqslant\|x-y\|_{\infty}$.

A standard assumption to make on stochastic processes with paths in $D$ is that the process has no fixed points of discontinuity. That is, $\mu\{x \in D: x$ is discontinuous at $\left.t_{0}\right\}=0$ for each fixed $t_{0} \in[0,1]$. In the next theorem we make this assumption and also assume that $\int\|x\|_{\infty}^{2} \mu(d x)<\infty$. Since $\|x\|_{\infty}=d(0, x)$, $\|\cdot\|_{\infty}$ is measurable on $D$. As $\mu \sim \nu$ where $\nu(d x)=\exp \left\{-\|x\|_{\infty}\right\} \mu(d x)$, this latter assumption is not a real limitation.

THEOREM 4.2. Let $\mu$ be a probability measure on $D$ with the Skorokhod topology. Assume that: 
(i) $\int\|x\|_{\infty}^{2} \mu(d x)<\infty$, and

(ii) $\mu\left\{x \in D\right.$ : $x$ is discontinuous at $\left.t_{0}\right\}=0$ for each fixed $t_{0}$ in $[0,1]$.

Let $Z$ be defined as above and let $V$ be the completion of $Z$ with respect to the norm $\|\nu\|^{2}=\int|\nu(x)|^{2} \mu(d x)$. Let $T: Z \rightarrow V$ be the natural inclusion and let $\phi: D \rightarrow Z^{*}$ be the natural imbedding. Then we have the following conclusions:

(1) $T$ and $T^{*}$ are continuous, compact maps.

(2) $\phi\left(S_{\mu}^{c}\right) \subseteq T^{*}\left(V^{*}\right)$.

(3) Suppose $C \subseteq S_{\mu}^{c}$ is convex, balanced, closed and bounded. Then $C$ is compact.

PROOF. Since $\|\nu\|_{\mu}^{2} \leqslant\|\nu\|^{2} \int\|x\|_{\infty}^{2} \mu(d x), T$ (and hence $T^{*}$ ) is continuous. Now suppose that $\left\{\nu_{n}\right\} \subseteq Z$ and $\left\|\nu_{n}\right\| \leqslant 1$. By going to a subsequence and re-indexing, we may assume that $\nu_{n}(x) \rightarrow \nu(x)$ for every continuous function $x$, where $\nu$ is a finite measure on $[0,1]$. Now,

$$
\begin{aligned}
\int_{D}\left|\nu_{n}(x)\right|^{2} \mu(d x) & =\int_{D}\left[\int_{0}^{1} \int_{0}^{1} x(s) x(t) \nu_{n}(d s) \nu_{n}(d t)\right] \mu(d x) \\
& =\int_{0}^{1} \int_{0}^{1}\left[\int_{D} x(s) x(t) \mu(d x)\right] \nu_{n}(d s) \nu_{n}(d t) .
\end{aligned}
$$

Now, $(s, t) \rightarrow \int_{D} x(s) x(t) \mu(d x)$ is continuous, since there are no fixed points of continuity. Hence (4.1) converges to

$$
\int_{0}^{1} \int_{0}^{1}\left[\int_{D} x(s) x(t) \mu(d x)\right] \nu(d s) \nu(d t)=\int_{D}|\nu(x)|^{2} \mu(d x) .
$$

Therefore $T$ and $T^{*}$ are compact. The second conclusion follows as in Theorem 1.2. Now suppose $C \subseteq S_{\mu}^{c}$ is convex, balanced, closed and bounded. Then $\phi(C)$ is convex, balanced and closed by Proposition 4.1 and bounded since $d(x, 0)=\|x\|_{\infty}$. As $\phi(C)$ is contained in the range of a compact operator, $\phi(C)$ is compact by Lemma 2.2. Hence $C=\phi^{-1}(\phi(C))$ is compact since $\left.\phi^{-1}\right|_{\phi(D)}$ is continuous.

\section{REFERENCES}

1. R. M. Dudley, Singular translates of measures on linear spaces, Z. Wahrscheinlichkeitstheorie und Verw. Gebiete 3 (1964), 128-137. MR 29 \#6529.

2. R. M. Fortet and E. Mourier, Les fonctions aléatoires comme éléments aléatoires dans les espaces de Banach, Studia Math. 15 (1955), 62-79. MR 19, 1202.

3. J. Hoffmann-Jprgensen and G. Pisier, The law of large numbers and the central limit theorem in Banach spaces (preprint).

4. J. L. Kelley and I. Namioka, Linear topological spaces, University Ser.in Higher Math., Van Nostrand, Princeton, N. J., 1963. MR 29 \#3851.

5. J. D. Kuelbs, Gaussian measures on a Banach space, J. Functional Analysis 5 (1970), 354-367. MR 41 \#4639.

6. J. Lindenstrauss and L. Tzafriri, Classical Banach spaces, Lecture Notes in Math., vol. 338, Springer-Verlag, Berlin and New York, 1973. 
7. A. Pietsch, Absolut p-summierende Abbildungen in normierten Räumen, Studia Math. 28 (1966/67), 333-353. MR 35 \#7162.

8. A. P. Robertson and W. Robertson, Topological vector spaces, Cambridge Univ. Press, New York, 1973.

9. V. N. Sudakov, On the characterization of quast-invariance of measures in Hilbert space, Uspehi Mat. Nauk 18 (1963), no. 1 (109), 188-190. (Russian) MR 26 \#6740.

10. Dao-Xing Xia (Hsia Tao-hsing), Measure and integration theory on infinite-dimensional spaces. Abstract harmonic analysis, Pure and Appl. Math., vol. 48, Academic Press, New York, 1972. MR 46 \#9281.

11. J. Zinn, Admissible translates of stable measures, Studia Math. 54 (1975), 245257.

DEPARTMENT OF MATHEMATICS, UNIVERSITY OF MASSACHUSETTS, AMHERST, MASSACHUSETTS 01002 